\title{
Using Cyber-Infrastructure for Dynamic Data Driven Laser Treatment of Cancer
}

\author{
C. Bajaj ${ }^{1}$, J.T. Oden ${ }^{1}$, K.R. Diller ${ }^{2}$, J.C. Browne ${ }^{1}$, J. Hazle ${ }^{3}$, I. Babuška ${ }^{1}$, \\ J. Bass ${ }^{1}$, L. Bidaut ${ }^{3}$, L. Demkowicz ${ }^{1}$, A. Elliott ${ }^{3}$, Y. Feng ${ }^{1}$, D. Fuentes ${ }^{1}$, \\ B. Kwon ${ }^{1}$, S. Prudhomme ${ }^{1}$, R.J. Stafford ${ }^{3}$, and Y. Zhang ${ }^{1}$ \\ ${ }^{1}$ Institute for Computational Engineering and Sciences, \\ ${ }^{2}$ Department of Biomedical Engineering, \\ The University of Texas at Austin, Austin TX 78712, USA \\ \{oden, babuska, bass, leszek, feng, fuentes, serge, jessica\}@ices.utexas.edu, \\ kdiller@mail.utexas.edu, \{bajaj, browne, juneim\}@cs.utexas.edu \\ ${ }^{3}$ University of Texas M.D. Anderson Cancer Center, \\ Department of Diagnostic Radiology, Houston TX 77030, USA \\ \{jhazle, jstafford, Andrew.Elliott, Luc.Bidaut\}@di.mdacc.tmc.edu \\ http://dddas.ices.utexas.edu
}

\begin{abstract}
Hyperthermia based cancer treatments are used to increase the susceptibility of cancerous tissue to subsequent radiation or chemotherapy treatments, and in the case in which a tumor exists as a well-defined region, higher intensity heat sources may be used to ablate the tissue. Utilizing the guidance of real-time treatment data while applying a laser heat source has the potential to provide unprecedented control over the outcome of the treatment process $6[12$. The goals of this work are to provide a working snapshot of the current system architecture developed to provide a real-time finite element solution of the problems of calibration, optimal heat source control, and goal-oriented error estimation applied the equations of bioheat transfer and demonstrate that current finite element technology, parallel computer architecture, peer-to-peer data transfer infrastructure, and thermal imaging modalities are capable of inducing a precise computer controlled temperature field within the biological domain.
\end{abstract}

\section{Introduction}

Thermal therapies delivered under various treatment modalities permit a minimally invasive and effective cancer treatment that eradicates the disease, maintains functionality of infected organs, and minimizes complications and relapse. The physical basis for thermal therapies is that exposing cells to temperatures outside their natural environment for certain periods of time can damage and even destroy the cells. However, one of the limiting factors in all forms of thermal therapies, including cryotherapy, microwave, radio-frequency, ultrasound, and laser, is the ability to control the energy deposition to prevent damage to adjacent healthy tissue 13 . 


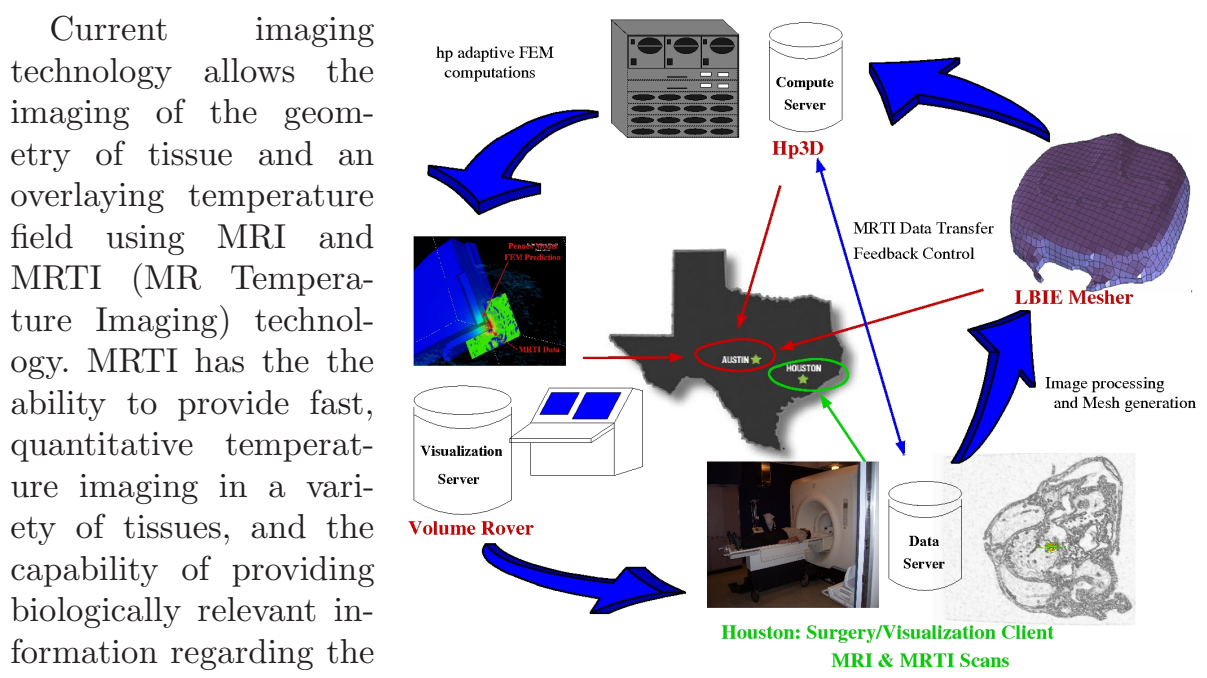

extent of injury immediately following a thermal therapy 4. Image guidance [1215] has the potential to facilitate Fig. 1. Schematic of the peer to peer communication architecture used to control the laser treatment process. Feedback control is achieved through the continual interaction of the data, compute, and visualization modules. unprecedented control over bioheat transfer by providing real time treatment monitoring through temperature feedback during treatment delivery. A similar idea using ultrasound guided cryotherapy has been studied and shows good results 13 .

The ultimate goal of this work is to deliver a computational model of bioheat transfer that employs real-time, patient specific data and provides realtime high fidelity predictions to be used concomitantly by the surgeon in the laser treatment process. The model employs an adaptive hp-finite element approximation of the nonlinear parabolic Pennes equation and uses adjoint-based algorithms for inverse analysis, model calibration, and adaptive control of cell damage. The target diseases of this research are localized adenocarcinomas of the breast, prostate, cerebrum, and other tissues in which a well-defined tumor may form. The algorithms developed also provide a potentially viable option to treat other parts of the anatomy in patients with more advanced and aggressive forms of cancer who have reached their limit of radiation and chemotherapy treatment.

\section{Software Architecture}

A schematic of the software architecture embedded in the control loop is shown in Figure 1. Figure 2 illustrates the main software modules and communication methods between software modules. Multiple client-server applications utilizing a remote procedure calling protocol connect the actual laboratory at M.D. Anderson Cancer Center in Houston, TX to the computing and visualization center 
in Austin, TX. Prior to treatment, the LBIE Mesher 11 uses MRI data to generate a finite element mesh of the patient-specific biological domain. Goal-oriented estimation and adap-

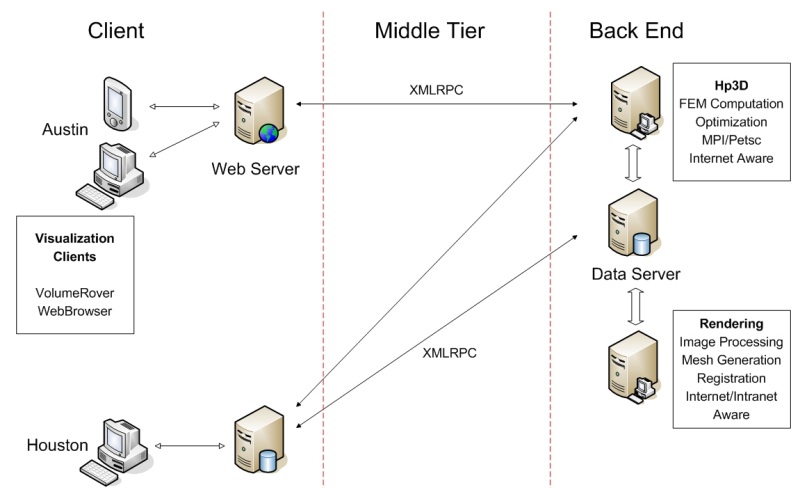

Fig. 2. Three Tier Cyber-software architecture. Computation, data transfer, and visualization are done on the backend compute nodes. A middle tier of XMLRPC connections connects the backend to the visualization clients in both Austin \& Houston. tion is used to optimize the mesh to a particular quantity of interest 9. The tool then proceeds to solve an optimal control problem, wherein the laser parameters (location of optical fiber, laser power, etc.) are controlled to eliminate/sensitize cancer cells, minimize damage to healthy cells, and control Heat Shock Protein (HSP) expression. Upon initiation of the treatment process, the compute server employs real-time MRI data to co-register the computational domain and MRTI data is used to calibrate the bioheat transfer model to the biological tissue values of the patient. As the data server, in Houston, delivers new data intermittently to the client, in Austin, computation is compared to the measurements of the real-time treatment and an appropriate course of action is chosen according to the differences seen. A parallel computing paradigm built from the Petsc [2] software infrastructure is used to meet the demands of rapid calibration and adapting the computational mesh and models to control approximation and modeling error. Volume Rover ${ }^{1}$ [ is used to achieve
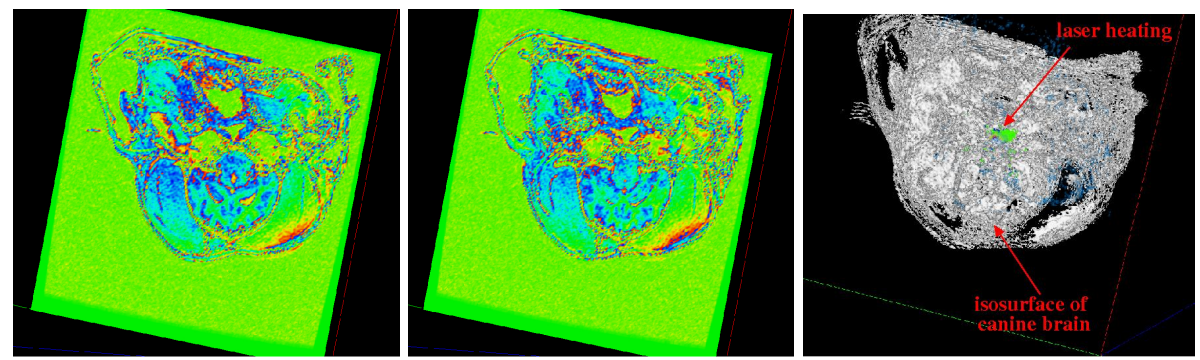

Fig. 3. Selected Slices of Canine MRI Brain Data, used for mesh generation. MRTI thermal data and Iso-surface visualization of Canine MRI Brain Data illustrate the location of heating $[8]$.

\footnotetext{
${ }^{1}$ Software available at: http://cvcweb.ices.utexas.edu/cvc
} 
efficient visualization of the volumetric MRI and thermal MRTI images simultaneously with the finite element prediction. From a computational point of view, the orchestration of a successful laser treatment is to solve the problems of coregistration, calibration, optimal control, and mesh refinement invisibly to the surgeon, and merely provide the surgeon with an interface to the optimal laser parameters and visualization of the computational prediction of the treatment treatment.

\section{Image Segmentation, Meshing, and MRTI-Registration}

Figure 4 shows a quality hexahedral mesh obtained for finite element simulations from a set of MRI data (256x256x34 voxels) of a canine brain, Figure 3. The field view of the MRI images was $200 \mathrm{~mm} \times 200 \mathrm{~mm}$ with each image spaced $1 \mathrm{~mm}$ apart. First, the image processing techniques, available in Volume Rover [1, were used to improve the quality of imaging data. Contrast enhancing techniques improved the contrast and anisotropic and bilateral diffusion [3] removed noise. Two dimensional segmentation was performed via a manual tracing of boundaries on each image slice, and the stack of contours tiled to form an initial water-tight triangulated surface. Three dimensional segmentation [1] could not be used because of the anisotropy in the imaging data. After the geometric model was obtained, geometric flow smoothed the geomet-

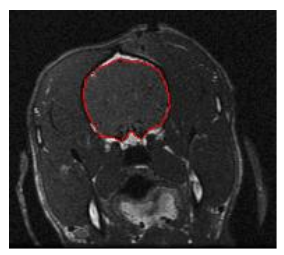

(a)

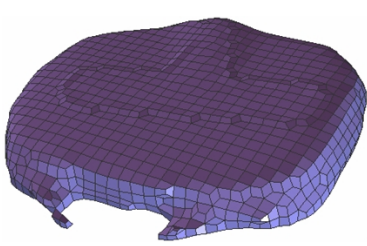

(c)

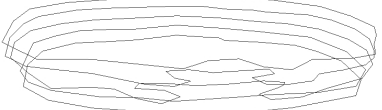

(b)

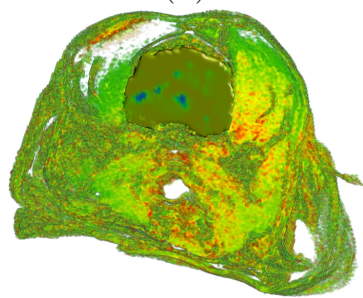

(d)

Fig. 4. Canine brain data. (a) Segmentation of the canine brain boundaries from the transverse 34-slice stack of $256 \times 256$ MRI data. A single slice is shown in gray-scale intensities, with the segmented boundary in red. (b) stack of $2 \mathrm{D}$ contours obtained from segmentation. (c) 8820 hexahedral mesh elements, Jacobian quality $>.05$. (d) combined volume visualization of the $256 \times 256 \times 32$ MRI data of a canine head, with the embedded subset of hexahedral finite element mesh of the segmented canine brain.

volumetric map using the signed distance function method was created. The hexahedral mesh was generated using an octree-based isocontouring method. Geometric flow [17, pillowing [7] and the optimization method were used to improve the mesh quality. The constructed hexahedral mesh has two important 
properties: good aspect ratios and there is at most one face lying on the boundary for each element.

The day of treatment, a FFT-based technique is used to register the finite element mesh to the current position of the patient. The registration software has been rigorously tested against a suite of validation experiments using phantom materials. The phantom materials are fabricated with two materials of contrasting image density in which an inner smaller object is placed asymmetrically within the larger object. The materials are composed of $2 \%$ agar gel and at least three $2 \mathrm{~mm}$ nylon beads are introduced as fiducials. The suite of data consists of several 3D images of incremental translational and rotational rigid body motions of the phantom material as well as images of incremental deformation of the phantom material. The data is provided for the image registration community from the DDDAS project webpag 2 .

The final image processing step is to overlay the MRTI thermal data onto the finite element mesh. A median and Deriche filter are used to remove the inherent noise from the MRTI data, Figure 5. The filtered MRTI data is interpolated onto the finite element solution space. The order of interpolation is determined by the order of the mesh.

\section{Calibration, Optimal Control, and Error Estimation}

Pennes model [10] has been shown [51614 to provide very accurate prediction of bioheat transfer and is used as the basis of the finite element prediction. The control paradigm involves three major problems: calibration of the Pennes bioheat transfer model to patient specific MRTI data, optimal positioning and power supply of the laser heat source, and computing goal oriented error estimates. During the laser treatment process, all three problems are solved in tandem by separate groups of processors communicating amongst each other as needed. The variational form of the governing Pennes bioheat transfer model is as follows:

$$
\begin{aligned}
& \text { Given a set of model, } \beta \text {, and laser, } \eta \text {, parameters, } \\
& \text { Find } u(\mathbf{x}, t) \in \mathcal{V} \equiv H^{1}\left([0, T], H^{1}(\Omega)\right) \text { s.t. } \\
& \qquad B(u, \beta ; v)=F(\eta ; v) \quad \forall v \in \mathcal{V}
\end{aligned}
$$

where the explicit functional dependence on the model parameters, $\beta$, and laser parameters, $\eta=\left(P(t), \mathbf{x}_{0}\right)$, are expressed as follows

$$
\begin{aligned}
B(u, \beta ; v)=\int_{0}^{T} \int_{\Omega} & {\left[\rho c_{p} \frac{\partial u}{\partial t} v+k(u, \beta) \nabla u \cdot \nabla v+\omega(u, \beta) c_{b l o o d}\left(u-u_{a}\right) v\right] d x d t } \\
+ & \int_{0}^{T} \int_{\partial \Omega_{C}} h u v d A d t+\int_{\Omega} u(\mathbf{x}, 0) v(\mathbf{x}, 0) d x
\end{aligned}
$$

${ }^{2}$ Project Website: dddas.ices.utexas.edu 


$$
\begin{aligned}
F(\eta ; v)= & \int_{0}^{T} \int_{\Omega} 3 P(t) \mu_{a} \mu_{t r} \frac{\exp \left(-\mu_{e f f}\left\|\mathbf{x}-\mathbf{x}_{0}\right\|\right)}{4 \pi\left\|\mathbf{x}-\mathbf{x}_{0}\right\|} v d x d t \\
& +\int_{0}^{T} \int_{\partial \Omega_{C}} h u_{\infty} v d A d t-\int_{0}^{T} \int_{\partial \Omega_{N}} \mathcal{G} v d A d t+\int_{\Omega} u^{0} v(\mathbf{x}, 0) d x \\
& \mu_{t r}=\mu_{a}+\mu_{s}(1-\gamma) \quad \mu_{e f f}=\sqrt{3 \mu_{a} \mu_{t r}}
\end{aligned}
$$

Here $k\left[\frac{\mathrm{J}}{\mathrm{s} \cdot \mathrm{m} \cdot \mathrm{K}}\right]$ and $\omega\left[\frac{\mathrm{kg}}{\mathrm{s} \mathrm{m}^{3}}\right]$ are bounded functions of $u, c_{p}$ and $c_{b l o o d}$ are the specific heats, $u_{a}$ the arterial temperature, $\rho$ is the density, and $h$ is the coefficient of cooling. $P$ is the laser power, $\mu_{a}, \mu_{s}$ are laser coefficients related to laser wavelength and give probability of absorption of photons by tissue, $\gamma$ is the anisotropy factor, and $\mathbf{x}_{0}$ is the position of laser photon source. Constitutive model data and details of the optimization process are given in 811].

\section{Data Transfer, Visualization, and Current Results}

Conventional data transfer methods and software rendering visualization tools pose a major bottleneck in developing a laser treatment paradigm in which high performance computers control the bioheat data transferred from a remote site. The data transfer problem is addressed through the use of client-server applications that use a remote procedure calling protocol to transfer data directly between physical memory instead of incurring the overhead of a writing to disk and transferring data. Volume Rover [1] is able to achieve high performance interactive visualization through the use of modern programmable graphics hardware to provide combined geometry and volume rendering displays, Figure 4 . Software rendering is limited by the memory and processor.

Computational time used to advance the Pennes model equations forward in time is not a bottleneck. Computations are done at the Texas Advanced Computing Center on a Dual-Core Linux Cluster. Each node of the cluster contains two Xeon Intel Duo-Core 64-bit processors (4 cores in all) on a single board, as an SMP unit. The core frequency is $2.66 \mathrm{GHz}$ and supports 4 floating-point operations per clock period. Each node contains 8GB of memory. The average execution times of a representative 10 second simulation is approximately 1 second, meaning that in a real time 10 second span Pennes model can predict out to more than a minute. Equivalently, in a 10 second time span, roughly 10 corrections can be made to calibrate the model coefficients or optimize the laser parameters.

The typical time duration of a laser treatment is about five minutes. During a five minute span, one set of MRTI data is acquired every 6 seconds. The size of each set of MRTI data is $\approx 330 \mathrm{kB}(256 \times 256 \times 5$ voxels). Computations comparing the predictions of Pennes model to experimental MRTI taken from a canine brain show very good agreement, Figure 5. A manual craniotomy of a canine skull was preformed to allow insertion of an interstitial laser fiber. A finite element mesh of the biological domain generated from the MRI data is shown in Figure 4 . The mesh consists of 8820 linear elements with a total of 


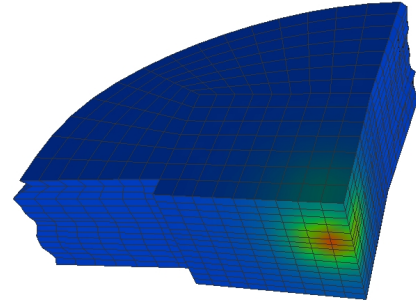

(a)

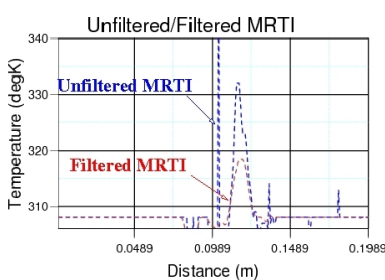

(b)

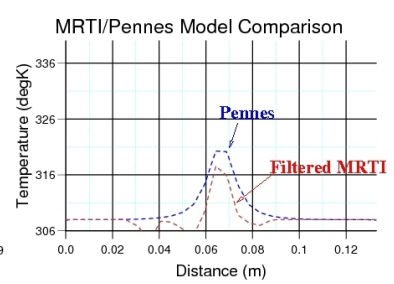

(c)

Fig. 5. (a) Contours of Pennes model prediction overlayed onto the finite element mesh. (b),(c) Simultaneous cutline comparison of Pennes model prediction, Filtered MRTI data, and Unfiltered MRTI data. Cutline taken through laser source.

9872 degrees of freedom. MRTI thermal imaging data was acquired in the form of five two dimensional 256x256 pixel images every six seconds for 120 time steps. The spacing between images was $3.5 \mathrm{~mm}$. The MRTI data was filtered then projected onto the finite element mesh. Figure 5 shows a cutline comparison between the MRTI data and the predictions of Pennes model. It is observed that the results delivered by the computational Pennes model slightly over diffuses the heat profile peaks compared to measured values. However, at early times the maximum temperature value is within $5 \%$ of the MRTI value.

\section{Conclusions}

Results indicate that reliable finite element model simulations of hyperthermia treatments can be computed, visualized, and provide feedback in the same time span that the actual therapy takes place. Combining these prediction capabilities with an understanding of HSP kinetics and damage mechanisms at the cellular and tissue levels due to thermal stress will provide a powerful methodology for planning and optimizing the delivery of hyperthermia therapy for cancer treatments.

The entire closed control loop in currently being tested on agar and ex-vivo tissue samples in preparation for the first real time computer guided laser therapy, which is anticipated within the upcoming year. The culmination of adaptive hp-finite element technology implemented on parallel computer architectures, modern data transfer and visualization infrastructure, thermal imaging modalities, and cellular damage mechanisms to provide cancer treatment tool will be a significant achievement in the field of computational science.

Acknowledgments. The research in this paper was supported in part by the National Science Foundation under grants CNS-0540033, IIS-0325550, and NIH Contracts P20RR0206475, GM074258. The authors also acknowledge the important support of DDDAS research by Dr. Frederica Darema of NSF. 


\section{References}

1. C. Bajaj, Z. Yu, and M. Aue. Volumetric feature extraction and visualization of tomographic molecular imaging. Journal of Structural Biology, 144(1-2):132-143, October 2003.

2. Satish Balay, William D. Gropp, Lois C. McInnes, and Barry F. Smith. Petsc users manual. Technical Report ANL-95/11 - Revision 2.1.5, Argonne National Laboratory, 2003.

3. W. Jiang, M. Baker, Q. Wu, C. Bajaj, and W. Chiu. Applications of bilateral denoising filter in biological electron microscopy. Journal of Structural Biology, 144:Issues 1-2:114-122, 2003.

4. M. Kangasniemi et al. Dynamic gadolinium uptake in thermally treated canine brain tissue and experimental cerebral tumors. Invest. Radiol., 38(2):102-107, 2003.

5. J. Liu, L. Zhu, and L. Xu. Studies on the three-dimensional temperature transients in the canine prostate during transurethral microwave thermal therapy. J. Biomech. Engr, 122:372-378, 2000.

6. R. J. McNichols et al. MR thermometry-based feedback control of laser interstitial thermal therapy at $980 \mathrm{~nm}$. Lasers Surg. Med., 34(1):48-55, 2004.

7. S. A. Mitchell and T. J. Tautges. Pillowing doublets: refining a mesh to ensure that faces share at most one edge. In Proc. 4th International Meshing Roundtable, pages pages 231-240, 1995 .

8. J. T. Oden, K. R. Diller, C. Bajaj, J. C. Browne, J. Hazle, I. Babuška, J. Bass, L. Demkowicz, Y. Feng, D. Fuentes, S. Prudhomme, M. N. Rylander, R. J. Stafford, and Y. Zhang. Dynamic data-driven finite element models for laser treatment of prostate cancer. Num. Meth. PDE, accepted.

9. J. T. Oden and S. Prudhomme. Goal-oriented error estimation and adaptavity for the finite element method. Computers and Mathematics with Applications, 41(5-6):735-756, 2001.

10. H. H. Pennes. Analysis of tissue and arterial blood temperatures in the resting forearm. J. Appl. Physiol., 1:93-122, 1948.

11. M. N. Rylander, Y. Feng, J. Zhang, J. Bass, Stafford R. J., J. Hazle, and K. Diller. Optimizing hsp expression in prostate cancer laser therapy through predictive computational models. J. Biomed Optics, 11:4:041113, 2006.

12. R. Salomir et al. Hyperthermia by MR-guided focused ultrasound: accurate temperature control based on fast MRI and a physical model of local energy deposition and heat conduction. Magn. Reson. Med., 43(3):342-347, 2000.

13. K. Shinohara. Thermal ablation of prostate diseases: advantages and limitations. Int. J. Hyperthermia, 20(7):679-697, 2004.

14. J.W. Valvano and et al. An isolated rat liver model for the evaluation of thermal techniques to measure perfusion. ASME J. Biomech. Eng., 106:187-191, 1984.

15. F. C. Vimeux et al. Real-time control of focused ultrasound heating based on rapid MR thermometry. Invest. Radiol., 34(3):190-193, 1999.

16. L. Xu, M.M. Chen, K.R. Holmes, and H. Arkin. The evaluation of the pennes, the chen-holmes, the weinbaum-jiji bioheat transfer models in the pig kidney vortex. ASME HTD, 189:15-21, 1991.

17. Y. Zhang, C. Bajaj, and G. Xu. Surface smoothing and quality improvement of quadrilateral/hexahedral meshes with geometric flow. In Proceedings of 14 th International Meshing Roundtable, volume 2, pages 449-468., 2005. 